\title{
Desempenho de frangos de corte alimentados com glicerina pura
}

\section{Performance of broiler fed pure glycerine}

\author{
Dássia Daiane de Oliveira ${ }^{1}$; João Waine Pinheiro ${ }^{2 *}$; Alexandre $\mathrm{Oba}^{2}$; \\ Nilva Aparecida Nicolao Fonseca ${ }^{3}$
}

\begin{abstract}
Resumo
Dois experimentos foram realizados com objetivo avaliar a glicerina pura (GLI) em rações de frangos de corte. O primeiro experimento consistiu de um ensaio de metabolismo em que foi utilizado o método de coleta total de excretas, com 96 frangos, machos, de 17 a 25 dias de idade, submetidos a dois tratamentos (ração referência e ração teste), sendo que a GLI substituiu a ração referência em $60 \mathrm{~g} / \mathrm{kg}$. A energia metabolizável aparente e a energia metabolizável aparente corrigida pelo balanço de nitrogênio foram de 4.015 e $3.911 \mathrm{kcal} / \mathrm{kg}$ na matéria natural, respectivamente. No segundo experimento foram utilizados 480 pintinhos, submetidos a 10 tratamentos constituídos de uma ração testemunha ( $0 \mathrm{~g} / \mathrm{kg}$ GLI) e da combinação de três inclusões de GLI na ração $(40,80$ e $120 \mathrm{~g} / \mathrm{kg})$ e três idades de início do seu fornecimento $(6,20$ e 34 dias). Os parâmetros avaliados foram ganho de peso, o consumo de ração e a conversão alimentar. O uso da glicerina pura na ração como fonte de energia deve levar em consideração a idade dos frangos, podendo ser incluída em até $120 \mathrm{~g} / \mathrm{kg}$ dos 20 aos 41 dias de idade.
\end{abstract}

Palavras-chave: Alimento alternativo, biodiesel, energia metabolizável, nutrição

\begin{abstract}
Two experiments evaluated the pure glycerin in broiler chicken diets. Experiment 1 was a metabolism test using total feces sampling method with 96 male chickens aging from 17 to $25 \mathrm{~d}$ when animals were fed on two treatments: $\operatorname{diet} 1=$ no glycerin and $\operatorname{diet} 2=60 \mathrm{~g} / \mathrm{kg}$ of glycerin. The apparent metabolized energy measured $4015 \mathrm{kcal} / \mathrm{kg}$ and the apparent metabolized corrected for nitrogen balance was $3911 \mathrm{kcal} / \mathrm{kg}$. Experiment 2 evaluated weight gains, feed intake and feed conversion in 480 chicks at 6,20 and $34 \mathrm{~d}$ old fed on diets with $0,40,80$ and $120 \mathrm{~g} / \mathrm{kg}$ of glycerin. The results indicate that pure glycerin in chicken diets, as a source of energy must take into consideration the age of the animals and it may be added up to $120 \mathrm{~g} / \mathrm{kg}$, from 20 to $41 \mathrm{~d}$ of age.
\end{abstract}

Key words: Alternative food, biodiesel, metabolizable energy, nutrition

${ }^{1}$ Zootecnista M.e em Ciência Animal. Universidade Estadual de Londrina, UEL, Londrina, PR. E-mail: dassiaoliveira@yahoo.com.br

2 Profs. Drs. do Dept ${ }^{\circ}$ de Zootecnia, UEL, Londrina, PR. E-mail: jwaine@uel.br; oba@uel.br

${ }^{3}$ Prof $^{\mathrm{a}} \mathrm{Dr}^{\mathrm{a}}$ Aposentada do Dept ${ }^{\mathrm{o}}$ de Zootecnia, UEL, Londrina, PR. E-mail: nilva@uel.br

* Autor para correspondência 


\section{Introdução}

A glicerina é o principal subproduto originado do processo de produção de biodiesel, através da transesterificação, sendo que para cada litro produzido são gerados cerca de cem mililitros de glicerina bruta.

Tradicionalmente a glicerina após ser purificada é utilizada na indústria cosmética, farmacêutica e alimentar, mas a crescente produção de biodiesel tem provocado aumentos significativos na sua disponibilização, gerando incertezas a respeito do seu destino, uma vez que a sua oferta é muito maior que a demanda. Assim, se torna necessária a viabilização de novos destinos para este subproduto.

A glicerina pode ser considerada carboidrato, se apresentando como fonte promissora de energia nas rações de frangos de corte (CERRATE et al., 2006; DOZIER et al., 2008). Em virtude do seu baixo peso molecular possui alta taxa de absorção intestinal e no fígado poderá ser convertida, via gliconeogênese, em glicose ou ser oxidada, por meio da glicólise e ciclo de Krebs, para a produção de energia (ROBERGS; GRIFFIN, 1998).

A glicerina pura caracteriza-se como alimento de alta energia, que é eficientemente utilizado pelas aves. De acordo com Barteczko e Kaminski (1999), o valor energético da glicerina pura é de $4.310 \mathrm{kcal} /$ $\mathrm{kg}$ de $\mathrm{EB}$, disponibilizando $4.200 \mathrm{kcal} / \mathrm{kg}$ de EMAn para os frangos de corte. Com relação à glicerina bruta, Dozier et al. (2008) determinaram em frangos de corte os valores de $3.621 \mathrm{kcal} / \mathrm{kg}$ de EMAn entre 7 e 10 dias de idade, $3.331 \mathrm{kcal} / \mathrm{kg}$ de EMAn entre 21 e 24 dias e $3.349 \mathrm{kcal} / \mathrm{kg}$ de EMAn entre 42 e 45 dias, sendo estes valores próximos aos da energia bruta (EB) determinada, que foi de $3.625 \mathrm{kcal} / \mathrm{kg}$, comprovando a sua alta disponibilidade energética.

A inclusão de glicerina pura nas rações de frangos em quantidades variando entre 50 e 100 $\mathrm{g} / \mathrm{kg}$ mostrou-se viável, sem afetar o desempenho (SIMON; BERGNER; SCHWABE, 1996). Menten,
Pereira e Racanicci (2008) também observaram bom desempenho dos frangos de corte alimentados com rações contendo $100 \mathrm{~g} / \mathrm{kg}$ de glicerina bruta. Estudos conduzidos por Cerrate et al. (2006) demonstraram que a glicerina bruta constitui produto passível de uso na alimentação de frangos, contudo concluíram que proporções superiores a 50 $\mathrm{g} / \mathrm{kg}$ na ração ocasionaram queda nos desempenhos dos mesmos, o que atribuíram ao menor consumo de ração pelos frangos causado pela dificuldade das rações fluírem nos comedouros.

O objetivo deste estudo foi determinar os valores energéticos da glicerina pura de origem animal e avaliar os seus efeitos sobre desempenho de frangos de corte.

\section{Material e Métodos}

Dois experimentos foram conduzidos no setor de avicultura da Universidade Estadual de Londrina. O primeiro experimento consistiu de estudo de digestibilidade, pelo método de coleta total de excretas (ALBINO et al., 1982), no qual foram utilizados 96 pintos de corte, machos, de 17 dias de idade, da linhagem Ross, alojados em 12 gaiolas dispostas em bateria metálica suspensa e distribuídos em delineamento inteiramente casualizado, com dois tratamentos e seis repetições de oito aves cada.

Inicialmente os frangos foram criados sobre cama de cepilho até 16 dias de idade, período em que foram submetidos aos manejos tradicionalmente empregados nas granjas comerciais, sendo alimentados com ração inicial à base de milho e farelo de soja e água à vontade. Aos 17 dias de idade, foram pesados individualmente e transferidos para as gaiolas de arame galvanizado, equipadas com bandejas coletoras de excretas revestidas com plástico, e providas de bebedouro tipo copo e comedouro tubular, com fornecimento de água e ração à vontade.

Os tratamentos experimentais consistiram 
de ração referência (Tabela 1), a base de milho e farelo de soja, formulada para atender às exigências nutricionais das aves, conforme as recomendações preconizadas por Rostagno et al. (2005), e ração teste, em que a glicerina pura substituiu, com base na matéria natural $(\mathrm{MN}), 60 \mathrm{~g} / \mathrm{kg}$ da ração referência (940 g/kg de dieta referência $+60 \mathrm{~g} / \mathrm{kg}$ de glicerina pura). A glicerina pura utilizada foi proveniente de sebo bovino, comercializada com o nome de Glicerina $525^{\circ}$, apresentando na sua composição o mínimo de $995 \mathrm{~g} / \mathrm{kg}$ de glicerol e no máximo $5 \mathrm{~g} /$ $\mathrm{kg}$ de água.

O estudo teve a duração de nove dias, sendo quatro dias para adaptação das aves às rações experimentais e às gaiolas e cinco dias para a coleta das excretas. O início e término da coleta das excretas foram determinados pelo aparecimento de fezes pigmentadas de vermelho, devido à adição de $10 \mathrm{~g}$ do marcador (óxido de ferro) $/ \mathrm{kg}$ de ração.

As excretas foram coletadas duas vezes ao dia, às 8:00 h e 16:00 h, a fim de evitar fermentação, embaladas em sacos plásticos identificados com o respectivo tratamento e repetição, pesadas e armazenadas a $-10^{\circ} \mathrm{C}$. A quantidade de ração consumida, bem como a quantidade de excreta produzida em cada unidade experimental, foi devidamente registrada.

Ao término do experimento, as excretas coletadas foram descongeladas por 24 horas, reunidas por repetições de cada tratamento e homogeneizadas. Em seguida, retiraram-se amostras de aproximadamente $500 \mathrm{~g}$, que após secagem em estufa ventilada a $55^{\circ} \mathrm{C}$ por 72 horas e resfriadas a temperatura ambiente por 12 horas, foram novamente pesadas e na sequência trituradas. Essas amostras, juntamente com as amostras das rações experimentais, foram analisadas e tiveram determinados os seus conteúdos de matéria seca e energia bruta, de acordo com as técnicas descritas por Silva e Queiroz (2002). A energia bruta da glicerina pura, das rações e das excretas foi determinada através do uso da bomba calorimétrica Ika, modelo C-200.
Tabela 1. Composição alimentar calculada da ração referência.

\begin{tabular}{lc}
\hline Ingrediente & $\begin{array}{c}\text { Valor } \\
(\mathrm{g} / \mathrm{kg} \text { de } \mathrm{MN})\end{array}$ \\
\hline Milho & 614,4 \\
Farelo de soja & 335,8 \\
Óleo de soja & 13,9 \\
Fosfato bicálcico & 17,8 \\
Calcário & 8,3 \\
Sal & 3,0 \\
Suplemento vitamínico-mineral ${ }^{1}$ & 4,0 \\
DL-metionina $(99 \%)$ & 1,1 \\
L- lisina $(79 \%)$ & 1,7 \\
\hline Total & 1000 \\
\hline Exigências atendidas & \\
\hline Energia metabolizável $(\mathrm{kcal} / \mathrm{kg})$ & 3.000 \\
Proteína bruta $(\mathrm{g} / \mathrm{kg})$ & 207,9 \\
Cálcio $(\mathrm{g} / \mathrm{kg})$ & 8,84 \\
Fósforo disponível $(\mathrm{g} / \mathrm{kg})$ & 4,42 \\
Metionina digestível $(\mathrm{g} / \mathrm{kg})$ & 5,34 \\
Metionina + cistina digestível $(\mathrm{g} / \mathrm{kg})$ & 8,14 \\
Lisina digestível $(\mathrm{g} / \mathrm{kg})$ & 11,46 \\
\hline
\end{tabular}

${ }^{1}$ Suplemento vitamínico-mineral (Composição por $\mathrm{kg}$ do produto): vit. A 1.500.000 UI; vit. D3 500.000 UI; vit. E 4.500 mg; vit. K3 200 mg; vit. B1 150 mg; vit. B2 $1.150 \mathrm{mg}$; vit. B6 $400 \mathrm{mg}$; vit. B12 $3.750 \mathrm{mcg}$; niacina $7.500 \mathrm{mg}$; pantotenato de cálcio $1.750 \mathrm{mg}$; ácido fólico $375 \mathrm{mg}$; biotina $6,25 \mathrm{mg}$; cloreto de colina $70.000 \mathrm{mg}$; ferro $7.500 \mathrm{mg}$; cobre $17.500 \mathrm{mg}$; manganês $15.000 \mathrm{mg}$; zinco $15.000 \mathrm{mg}$; iodo $300 \mathrm{mg}$; selênio $75 \mathrm{mg}$; antioxidante $25.000 \mathrm{mg}$; coccidiostático $15.000 \mathrm{mg}$; promotor de crescimento e eficiência alimentar $12.500 \mathrm{mg}$.

Fonte: Elaboração dos autores.

Os valores de energia metabolizável aparente (EMA) e aparente corrigida pelo balanço de nitrogênio (EMAn) da glicerina pura, foram determinados utilizando as equações propostas por Matterson et al. (1965).

$\mathrm{O}$ segundo experimento consistiu em avaliar o desempenho de frangos de corte de 6 a 41 dias de idade alimentados com GLI. Foram utilizados 480 frangos de corte machos, alojados em aviário convencional, dividido em 40 boxes de 1,50 $\mathrm{m}^{2}$ com piso recoberto com cepilho e equipados com comedouro tubular e bebedouro pendular. 
Aos seis dias de idade os pintinhos foram pesados e distribuídos nos boxes, em dez tratamentos experimentais, em delineamento experimental inteiramente casualizado, mantendose o peso médio do lote por unidade experimental, sendo que cada tratamento teve quatro repetições de 12 aves cada, totalizando 48 aves por tratamento.

Os tratamentos experimentais consistiram de combinação de níveis de inclusão de glicerina pura (GLI) nas rações, 0, 40, 80 e $120 \mathrm{~g} / \mathrm{kg}$, e idade de início do fornecimento destas rações $(6,20 \mathrm{e}$ 34 dias de idade até os 41 dias), totalizando dez tratamentos, sendo:

T1 - ração contendo $0 \mathrm{~g} / \mathrm{kg}$ de GLI de 6 a 41 dias de idade;

T2 - ração contendo $40 \mathrm{~g} / \mathrm{kg}$ de GLI na ração a partir de 6 dias de idade;

T3 - ração contendo $80 \mathrm{~g} / \mathrm{kg}$ de GLI na ração a partir de 6 dias de idade;

T4 - ração contendo $120 \mathrm{~g} / \mathrm{kg}$ de GLI na ração a partir de 6 dias de idade;

T5 - ração contendo $40 \mathrm{~g} / \mathrm{kg}$ de GLI na ração a partir de 20 dias de idade;

T6 - ração contendo $80 \mathrm{~g} / \mathrm{kg}$ de GLI na ração a partir de 20 dias de idade;

T7 - ração contendo $120 \mathrm{~g} / \mathrm{kg}$ de GLI na ração a partir de 20 dias de idade;

T8 - ração contendo $40 \mathrm{~g} / \mathrm{kg}$ de GLI na ração a partir de 34 dias de idade;

T9 - ração contendo $80 \mathrm{~g} / \mathrm{kg}$ de GLI na ração a partir de 34 dias de idade;
T10 - ração contendo $120 \mathrm{~g} / \mathrm{kg}$ de GLI na ração a partir de 34 dias de idade.

A ração controle foi fornecida aos frangos até a idade do início do recebimento das rações contendo GLI.

As rações experimentais (Tabela 2), para cada fase de desenvolvimento dos frangos, foram formuladas para atender as exigências nutricionais mínimas, preconizadas por Rostagno et al. (2005), sendo fornecidas à vontade. Foi considerado o valor da EMA da glicerina pura obtido no ensaio de digestibilidade.

Aos 6, 20, 34 e 41 dias de idade foram realizadas pesagens dos frangos e das rações para a obtenção dos dados referentes às características consumo de ração, ganho de peso e conversão alimentar.

As médias dos parâmetros estudados, obtidas aos 41 dias, foram submetidas à análise de variância e regressão através do programa SAEG (Sistema para análises estatísticas e genéticas, versão 9.0.). Foram realizados estudos para avaliar a interação entre os fatores tipo de ração (sem e com GLI) e idade de início do seu fornecimento $(6,20$ e 34 dias). Na ausência de interação entre esses fatores procedeu-se às análises dos mesmos de forma isolada. Assim, foram realizadas comparações entre as médias obtidas com a ração controle (sem GLI) e a ração contendo GLI. Foram feitos estudos de regressão para determinar a melhor idade de início da inclusão da GLI nas rações (6, 20 e 34 dias) e o melhor nível da sua inclusão (0, 40, 80 e 120 g/kg) dentro de cada idade. 


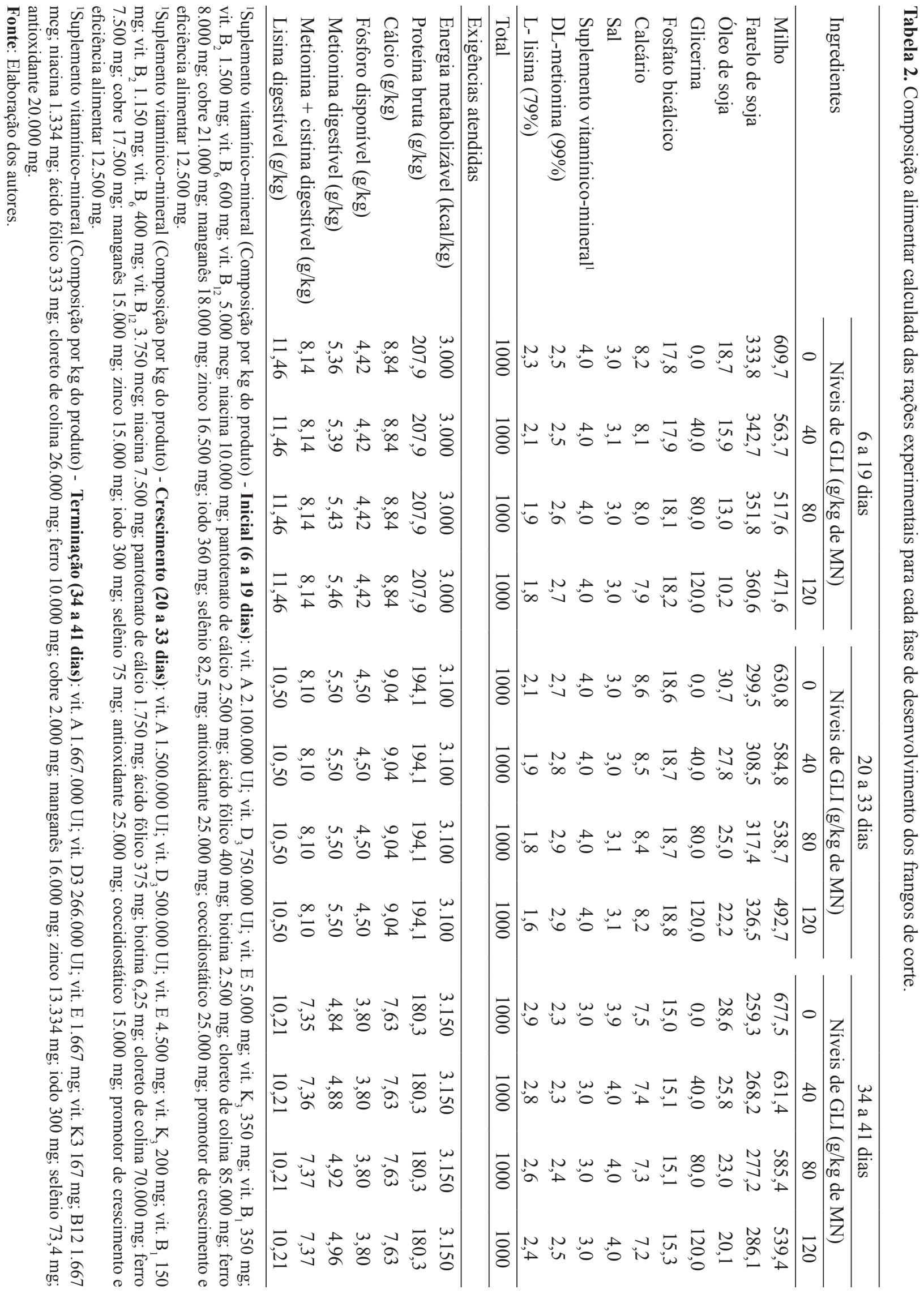




\section{Resultados e Discussão}

O valor de energia bruta $(\mathrm{EB})$ da glicerina purificada (GLI) foi de $4.567 \mathrm{kcal} / \mathrm{kg}$ de $\mathrm{MN}$, o de EMA foi de $4.015 \mathrm{kcal} / \mathrm{kg}$ de MN e a EMAn de 3.911 $\mathrm{kcal} / \mathrm{kg}$ de $\mathrm{MN}$, determinados em frangos de 21 a 25 dias de idade. De acordo com Nery et al. (2007), é normal os valores da EMAn serem inferiores aos da EMA, quando estes são determinados em aves em crescimento, pois nesta fase ocorre maior retenção de nitrogênio para a deposição de tecido muscular. Assim, é necessário corrigir os valores estimados de energia pelo balanço de nitrogênio.

O valor de EMA determinado $(4.015 \mathrm{kcal} /$ $\mathrm{kg}$ ) foi inferior ao citado por Kerr, Dozier III e Bregendahl (2007) em que os valores de EMA para a glicerina pura foram de 4.237 e 4.056 $\mathrm{Kcal} / \mathrm{kg}$, para as substituições de 50 e $100 \mathrm{~g} / \mathrm{kg}$ na ração, respectivamente. É importante destacar que a quantidade de substituição na ração teste e a idade dos animais constituem fonte de variação nas estimativas da energia metabolizável das glicerinas (DOZIER et al., 2008).

A EMAn (3.911 kcal $/ \mathrm{kg})$ determinada neste estudo foi superior aos $3.331 \mathrm{kcal} / \mathrm{kg}$ determinados por Dozier et al. (2008) em frangos de 21 a 24 dias de idade, alimentados com 0, 30, 60 e $90 \mathrm{~g} /$ $\mathrm{kg}$ de glicerina bruta e ao valor de $3.490 \mathrm{Kcal} / \mathrm{kg}$ de EMAn encontrado por Gianfelici (2009), para uma inclusão de $100 \mathrm{~g} / \mathrm{kg}$ de glicerina bruta na ração de frangos com 35 dias. O valor de EMAn determinado $(3.911 \mathrm{kcal} / \mathrm{kg})$ corresponde a $86 \%$ do valor de EB (4.567 kcal/ $\mathrm{kg}$ ), confirmando que a energia deste ingrediente tem alta disponibilidade. Vale salientar ainda que a diferença na energia metabolizável da glicerina observada neste estudo, em relação a outros estudos, pode ser devido à pureza da amostra, pois a concentração de glicerol e a presença de contaminantes (metanol, cloreto de sódio ou de potássio) são variáveis no subproduto, interferindo no seu metabolismo (LAMMERS et al., 2008).

Comparada com o milho, que constitui o alimento energético padrão em rações para frangos de corte, a glicerina pura pode ser considerada fonte de energia passível de utilização na alimentação, em virtude do seu bom teor de energia bruta, altamente metabolizável.

Os resultados de desempenho dos frangos utilizados no experimento 2 estão apresentados na Tabela 3, na qual se observa que não houve interação entre o tipo de ração e a idade de início do seu fornecimento. Analisando os fatores de forma isolada, observa-se que os mesmos não influenciaram $(\mathrm{P}>0,05)$ o consumo de ração, ganho de peso e conversão alimentar das aves nas idades estudadas.

O estudo das rações dentro de cada idade demonstrou que inclusão da GLI a partir dos seis dias de idade afetou a conversão alimentar, que apresentou resposta quadrática $(\mathrm{P}<0,05)$, expressa pela equação $\mathrm{Y}=1,6687-0,0008 \mathrm{X}+0,00001348 \mathrm{X}^{2}$, $\mathrm{R}^{2}=0,93$, com mínimo de $30 \mathrm{~g}$ de GLI/kg de ração, indicando que existe limitação do seu uso pelas aves, quando a GLI é fornecida a partir desta idade. Assim como neste experimento, Simon, Bergner e Schwabe (1996) ao avaliarem a inclusão de até 100 g GLI/kg de ração, também não encontraram efeitos no consumo de ração e no ganho de peso dos frangos, no entanto, Zoidis et al. (2010) observaram piora na conversão alimentar dos frangos alimentados com rações contendo níveis crescentes de glicerol (70, 140 e $210 \mathrm{~g} / \mathrm{kg}$ ) do $1^{\circ}$ ao $42^{\circ}$ dia de idade. 
Tabela 3. Médias dos parâmetros de desempenho dos frangos de corte obtidas aos 41 dias de idade.

\begin{tabular}{|c|c|c|c|}
\hline & Consumo de ração (kg) & Ganho de peso $(\mathrm{kg})$ & Conversão alimentar $(\mathrm{kg} / \mathrm{kg})$ \\
\hline \multicolumn{4}{|c|}{ Interação Tipo de ração x Idade } \\
\hline Valor P & 0,082 & 0,421 & 0,362 \\
\hline \multicolumn{4}{|c|}{ Tipo de ração } \\
\hline Sem GLI & 4,267 & 2,565 & 1,664 \\
\hline Com GLI & 4,286 & 2,539 & 1,689 \\
\hline Média & 4,284 & 2,541 & 1,687 \\
\hline EPM & 0,021 & 0,015 & 0,007 \\
\hline Valor P & 0,779 & 0,542 & 0,167 \\
\hline \multicolumn{4}{|c|}{ Idade de início de inclusão da GLI na ração } \\
\hline 6 dias & 4,321 & 2,533 & 1,707 \\
\hline 20 dias & 4,241 & 2,532 & 1,675 \\
\hline 34 dias & 4,296 & 2,550 & 1,686 \\
\hline Média & 4,286 & 2,538 & 1,689 \\
\hline EPM & 0,023 & 0,016 & 0,008 \\
\hline \multirow{2}{*}{$\begin{array}{ll}\text { Valor } \mathrm{P}^{1} \mathrm{~L} \\
\\
\mathrm{Q}\end{array}$} & 0,642 & 0,621 & 0,149 \\
\hline & 0,143 & 0,754 & 0,087 \\
\hline \multicolumn{4}{|c|}{ Efeito de regressão para os níveis de inclusão de GLI na ração $(\mathrm{g} / \mathrm{kg})$ aos 6 dias de idade } \\
\hline 0 & 4,267 & 2,565 & 1,664 \\
\hline 40 & 4,312 & 2,577 & 1,674 \\
\hline 80 & 4,286 & 2,559 & 1,676 \\
\hline 120 & 4,366 & 2,465 & 1,772 \\
\hline Média & 4,308 & 2,541 & 1,696 \\
\hline EPM & 0,023 & 0,018 & 0,013 \\
\hline Valor $\mathrm{P}^{1} \mathrm{~L}$ & 0,353 & 0,090 & 0,000 \\
\hline $\mathrm{Q}$ & 0,793 & 0,203 & 0,018 \\
\hline \multicolumn{4}{|c|}{ Efeito de regressão para os níveis de inclusão de GLI na ração $(\mathrm{g} / \mathrm{kg})$ aos 20 dias de idade } \\
\hline 0 & 4,267 & 2,565 & 1,664 \\
\hline 40 & 4,276 & 2,575 & 1,661 \\
\hline 80 & 4,167 & 2,523 & 1,652 \\
\hline 120 & 4,276 & 2,498 & 1,712 \\
\hline Média & 4,247 & 2,540 & 1,670 \\
\hline EPM & 0,034 & 0,017 & 0,011 \\
\hline Valor $\mathrm{P}^{1} \mathrm{~L}$ & 0,793 & 0,177 & 0,085 \\
\hline $\mathrm{Q}$ & 0,452 & 0,666 & 0,077 \\
\hline \multicolumn{4}{|c|}{ Efeito de regressão para os níveis de inclusão de GLI na ração $(\mathrm{g} / \mathrm{kg})$ aos 34 dias de idade } \\
\hline 0 & 4,267 & 2,565 & 1,664 \\
\hline 40 & 4,369 & 2,613 & 1,673 \\
\hline 80 & 4,370 & 2,610 & 1,675 \\
\hline 120 & 4,150 & 2,427 & 1,710 \\
\hline Média & 4,289 & 2,554 & 1,680 \\
\hline EPM & 0,037 & 0,029 & 0,006 \\
\hline Valor $\mathrm{P}^{1} \mathrm{~L}$ & 0,235 & 0,029 & 0,078 \\
\hline $\mathrm{Q}$ & 0,018 & 0,008 & 0,472 \\
\hline
\end{tabular}

${ }^{1}$ L e Q: efeitos de ordem linear e quadrática relativos à inclusão da glicerina na ração; EPM: erro padrão da média. Conversão alimentar aos 6 dias: $Y=1,6687-0,0008 X+0,00001348 X^{2}, R^{2}=0,93$.

Consumo de ração aos 34 dias: $Y=4,2607+0,0051699 X-0,00005035 X^{2}, R^{2}=0,98$.

Ganho de peso aos 34 dias: $Y=2,55845+0,0032796 X-0,000036011 X^{2}, R^{2}=0,96$.

Fonte: Elaboração dos autores. 
As rações contendo as diferentes concentrações de GLI quando fornecidas aos 20 dias de idade não afetaram $(\mathrm{P}>0,05)$ o consumo de ração, o ganho de peso e a conversão alimentar. Estes resultados se assemelham aos de Menten, Pereira e Racanicci (2008) que também encontraram semelhanças entre os desempenhos dos frangos alimentados com rações contendo $100 \mathrm{~g} / \mathrm{kg}$ de glicerina bruta em relação aos alimentados com uma ração testemunha, isenta do produto. Da mesma forma, Abd-Elsamee et al. (2010) verificaram que a glicerina bruta pode compor até $80 \mathrm{~g} / \mathrm{kg}$ das rações dos frangos, sem causar efeitos adversos no seu desempenho. Contudo, estes resultados discordam dos verificados por Guerra (2010), que ao pesquisar a inclusão de até $100 \mathrm{~g} / \mathrm{kg}$ de glicerina bruta mista $(950 \mathrm{~g} / \mathrm{kg}$ de origem vegetal e $50 \mathrm{~g} / \mathrm{kg}$ de origem animal) na ração dos frangos a partir de 21 dias de idade, verificou redução no consumo de ração e no ganho de peso e piora na conversão alimentar.

Os níveis crescentes de GLI nas rações ofertadas aos frangos a partir dos 34 dias de idade determinaram resposta quadrática $(\mathrm{P}<0,05)$ no consumo de ração $(\mathrm{Y}=$ $\left.4,2607+0,0051699 \mathrm{X}-0,00005035 \mathrm{X}^{2} \quad\left(\mathrm{R}^{2}=0,98\right)\right)$ com máximo em $51 \mathrm{~g} / \mathrm{kg}$, e no ganho de peso $(\mathrm{Y}=\quad 2,55845+0,0032796 \mathrm{X}-0,000036011 \mathrm{X} 2$ $\left.\left(\mathrm{R}^{2}=0,96\right)\right)$, com máximo em $46 \mathrm{~g} / \mathrm{kg}$, não tendo efeito $(\mathrm{P}>0,05)$ na conversão alimentar.

Uma provável explicação para este resultado é que o tempo em que os frangos ingeriram as rações experimentais (sete dias), provavelmente tenha sido insuficiente para a adaptação das enzimas intestinais a GLI, afetando negativamente o consumo de ração e o ganho de peso, nos seus níveis mais elevados de inclusão.

Os valores ótimos de inclusão da GLI na ração de frangos nesta fase de desenvolvimento estão próximos às $50 \mathrm{~g} / \mathrm{kg}$ estabelecidos por Simon, Bergner e Schwabe (1996).

\section{Conclusão}

A glicerina pura de origem animal caracteriza-se como fonte de energia (4.015 kcal $/ \mathrm{kg}$ de EMA) a ser utilizada na alimentação dos frangos de corte, contudo o seu uso está na dependência da idade destas aves e da quantidade a ser incluída na ração, podendo compor até $120 \mathrm{~g} / \mathrm{kg}$ de ração dos frangos dos 20 aos 41 dias de idade.

\section{Referências}

ABD-ELSAMEE, M. O.; ABDO, Z. M. A.; ELMANYLAWI, M. A. F.; SALIM, I. H. Use of crude glycerin in broiler diets. Egyptian Poultry Science, Alexandria, v. 30, n. 1, p. 281-295, 2010.

ALBINO, L. F. T.; FERREIRA, A. S.; FIALHO, E. T.; CESAR, S. S. Determinação dos valores de energia metabolizável e matéria seca aparentemente metabolizável de alguns alimentos. Revista Brasileira de Zootecnia, Viçosa, v. 11, n. 2, p. 207-220, 1982.

BARTECZKO, J.; KAMINSKI, J. The effect of glycerol and vegetable fat on some physiological índices of the blood and overfatness of broiler carcass. Annals Warsaw Agricultural University Animal Science, Warsaw, v. 36, p. 197-209, 1999.

CERRATE, S.; YAN, F.; WANG, Z.; COTO, C.; SACAKLI, P.; WALDROUP, P.W. Evaluation of glycerine from biodiesel production as a feed ingredient for broilers. International Journal of Poultry Science, Faisalabad, v. 5, n. 11, p. 1001-1007, 2006.

DOZIER III, W. A.; KERR, B. J.; CORZO, A.; KIDD, M. T.; WEBER, T. E.; BREGENDALS, K. Apparent metabolizable energy of glycerin for broiler chickens. Poultry Science, Savoy, v. 87, n. 2, p. 317-322, 2008.

GIANFELICI, M. F. Uso de glicerol como fonte de energia para frangos de corte. 2009. Dissertação (Mestrado em Zootecnia) - Universidade Federal do Rio Grande do Sul, Porto Alegre.

GUERRA, R. L. H. Glicerina bruta na alimentação de frangos de corte. 2010. Dissertação (Mestrado em Zootecnia) - Universidade Estadual de Maringá, Maringá.

KERR, B. J.; DOZIER III, W. A.; BREGENDAHL, K. Nutritional value of crude glycerin for nonruminants. In: ANNUAL CAROLINA SWINE NUTRITION CONFERENCE, 23., 2007, Raleigh, North Carolina. Proceedings...Raleigh, 2007. p. 6-8. Disponível em: $<$ http://biodieselfeeds.cfans.umn.edu/.../2007-Kerr- 
Nutritional $\% 20$ value $\% 20$ of $\% 20$ crude $\% 20$ glycerin $\% 20$ for\%20non-ruminan...>. Acesso em: 27 set. 2013.

LAMMERS, P. J.; KERR, B. J.; HONEYMAN, M. S.; STALDER, K.; DOZIER III, W. A.; WEBER, T. E.; KIDD, M. T.; BREGENDAHL, K. Nitrogen-corrected apparent metabolizable energy value of crude glycerol for laying hens. Poultry Science, Savoy, v. 87, n. 1, p. 104-107, 2008.

MATTERSON, L. D.; POTTER, L. M.; STUTUZ, N. W.; SINGSEN, E. P. The metabolizable energy of feed ingredients for chickens. Connecticut: Agricultural Experiment Station Research Report, University of Connecticut Storrs, 1965. 11 p.

MENTEN, J. F. M.; PEREIRA, P. W. Z.; RACANICCI, A. M. C. Avaliação da glicerina proveniente do biodiesel como ingrediente para rações de frangos de corte. In: CONFERÊNCIA APINCO DE CIÊNCIA E TECNOLOGIA AVÍCOLAS, 10., 2008, Santos. Anais... Campinas: Fundação APINCO de Ciência e Tecnologia Avícolas, 2008. p. 66.

NERY, L. R.; ALBINO, L. F. T.; ROSTAGNO, H. S.; CAMPOS, A. M. A.; SILVA, C. R. Valores de energia metabolizável de alimentos determinados com frangos de corte. Revista Brasileira de Zootecnia, Viçosa, v. 36, n. 5, p. 1354-1358, 2007.
ROBERGS, R.A.; GRIFFIN, S. E. Glycerol: biochemistry, pharmacokinetics and clinical and practical applications. Sports Medicine, v. 26, n. 3, p. 145-167, 1998.

ROSTAGNO, H. S.; ALBINO, L. F. T.; DONZELE, J. L.; GOMES, P. C.; OLIVEIRA, R. F.; LOPES, D. C.; FERREIRA, A. S.; BARRETO, S. L. T. Tabelas brasileiras para aves e suinos: composição de alimentos e exigências nutricionais. 2. ed. Viçosa: Universidade Federal de Viçosa, 2005. 186 p.

SILVA, D. J.; QUEIROZ, A. C. Análises de alimentos: métodos químicos e biológicos. 3. ed. Viçosa: Universidade Federal de Viçosa, 2002. 235 p.

SIMON, A.; BERGNER, H.; SCHWABE, M. Glycerol feed ingredient for broiler chickens. Archives of Animal Nutrition, Berlin, v. 49, n. 2, p. 103-112, 1996.

ZOIDIS, E.; PAPADOMICHELAKIS, G.; MOUNTZOURIS, K. C.; PAPPAS, A. C.; ARVANITI, A.; FEGEROS, K. Dietary glycerol level effects on performance traits, glycerol kinase gene expression and gut microbiota in broilers. In: ANNUAL MEETING, 60, Greece, 2010. Disponível em: <http://www.eaap. org/Crete/Papers/07_Zoidis.pdf. $>$. Acesso em: 27 set. 2013. 
